\title{
Compound stimulus control of operant, but not adjunctive, behavior
}

\author{
ROBERT J. HAMM, JOSEPH H. PORTER, and GERALD D. OSTER \\ Virginia Commonwealth University, Richmond, Virginia 23284
}

\begin{abstract}
Compound stimulus control of operant (barpress) and adjunctive (polydipsia) behaviors was investigated by training rats under a multiple schedule of food delivery. Four rats (operant group) were reinforced for barpressing during light $(\mathrm{L}+\overline{\mathrm{T}})$ or tone $(\overline{\mathrm{L}}+\mathrm{T})$. For half of the operant group, barpressing was not reinforced during no light and no tone $(\overline{\mathrm{L}}+\overline{\mathrm{T}})$, while the other half were not reinforced during light and tone $(\mathrm{L}+\mathrm{T})$. The four adjunctive behavior rats were each yoked to a rat in the operant group so that food pellets were delivered to the yoked rats at the same rate and during the same stimulus conditions. The adjunctive behavior recorded was the licking of a water bottle induced by the intermittent delivery of food. When a compound stimulus was presented that was composed exclusively of the light and tone elements that had been paired with food-pellet delivery and increased barpressing or licking, it controlled the highest response rate of all stimuli (additive summation) for the operant behavior but not for the adjunctive behavior.
\end{abstract}

Traditionally, behavior has been classified as either operant behavior or respondent behavior. However, a third behavioral class has been proposed. Falk (1971) has suggested that "when certain schedules induce extra, concurrent phenomena which are strong enough to sustain scheduled behavior in their own right, that these phenomena be called adjunctive behaviors" (p. 578). Adjunctive behaviors are characterized as being "induced" by a stimulus (usually a food pellet or some other reinforcer) as contrasted to respondent behaviors that are "elicited" or operant behaviors that are "emitted." Within a schedule of periodic food delivery, adjunctive behavior occurs when the probability of reinforcement is low (i.e., immediately postpellet); whereas, operant behavior occurs when reinforcement probability is high (i.e., prepellet). Staddon and Simmelhag (1971) have termed these adjunctive and operant behaviors as interim activities and terminal responses, respectively. More recently, Falk (1977) has further argued for consideration of adjunctive behaviors as a distinct behavioral class and has even proposed four adjunctive behavior "laws." In fact, the use of adjunctive behavior as a separate behavioral class has become more frequent in recent literature (e.g., Skinner, 1977; Staddon, 1977).

One line of research concerning adjunctive behaviors concerns the similarities and/or differences between adjunctive and operant behaviors. Porter and Allen (1977) have shown that positive behavioral contrast can be obtained with an adjunctive behavior (i.e., schedule-induced polydipsia) when the standard

Reprint requests should be sent to Robert J. Hamm, Department of Psychology, Virginia Commonwealth University, Richmond, Virginia 23284. multiple-schedule paradigm for operant behaviors (see Reynolds, 1961) is used. In addition, Cohen (1975) has demonstrated that schedule-induced drinking follows the matching law (de Villiers, 1977), which is another commonly observed characteristic of operant behaviors. These data suggest that adjunctive behaviors show some of the behavioral interactions that are observed with operant behaviors and that adjunctive and operant behaviors may be controlled by the same processes. If this is correct, the necessity of a third behavioral class is questionable (cf. Herrnstein, 1977). The purpose of the present experiment was to further explore the processes that control operant and adjunctive behaviors.

Additive summation is a well established phenomena in both operant and respondent conditioning (see Weiss, 1972), but it has not been investigated using an adjunctive behavior. Stimulus compounding involved in summation has frequently been studied by simultaneously presenting two stimuli that have previously been conditioned separately. If the simultaneous presentation of the two stimuli (i.e., a compound stimulus) controls a higher response rate than any of the separate stimuli, additive summation has been observed. A frequently used method to investigate additive summation is to use a three-component multiple schedule. Responding is maintained by reinforcement in the presence of either of two $\mathrm{SD}_{\mathrm{S}}$ and reduced through nonreinforcement during $S^{\Delta}$. Typically, the $S^{D}{ }_{S}$ are light (or more precisely, $\mathrm{L}+\overline{\mathrm{T}})$ and tone $(\overline{\mathrm{L}}+\mathrm{T})$, and $\mathrm{S}^{\Delta}$ is the absence of light and tone $(\overline{\mathrm{L}}+\overline{\mathrm{T}})$. When the two $\mathrm{S}^{\mathrm{D}} \mathrm{S}$ are simultaneously presented (i.e., the compound stimulus, $\mathrm{L}+\mathrm{T}$ ), it controls a higher response rate than either $S^{D}$ (additive summation).

The present experiment attempted to determine whether the processes that produce additive summation 
of an operant behavior will also produce summation of an adjunctive behavior. The adjunctive behavior chosen for study was schedule-induced polydipsia.

\section{METHOD}

\section{Subjects}

Subjects were eight female Sprague-Dawley rats between the ages of 90 and 120 days old. Ten days prior to the start of the experiment, subjects were reduced to $80 \%$ of their free-feeding weights and maintained at that level by restricting their daily ration of Purina Rat Chow. Water was continuously available in the home cages. Rats were randomly assigned to two groups of four.

\section{Apparatus}

Two Scientific Prototype (Model D700-1305) chambers were used. The interior dimensions of the chamber were $23 \times 21 \times 19 \mathrm{~cm}$. The front wall of the chamber had the following configuration: a response bar centered on the midline of the wall and $3 \mathrm{~cm}$ above the grid floor, a food-pellet cup located $4 \mathrm{~cm}$ from the left wall and $1 \mathrm{~cm}$ above the floor, a cue light $(28 \mathrm{~V} \mathrm{dc}) 6 \mathrm{~cm}$ above the response bar, and a hole $1.5 \mathrm{~cm}$ in diam. The hole provided access to a water bottle. The water bottle was mounted on the outside of the chamber so that the end of the drinking spout (2.4- $\mathrm{mm}$ aperture diameter) was flush with the front wall. Contact with the water tube was recorded by BRS contact relay (DR901/221-05). A Mallory Sonalert (Model SC 628-11) was centered on the left side wall. Solid state programming equipment was located in an adjacent room and controlled stimulus events and response recording.

\section{Procedure}

Preliminary training. Rats were placed in the chamber for four 1-h sessions with $5045-\mathrm{mg}$ food pellets in the food cup, and the amount of water consumed was recorded. All rats were then trained to approach and eat the $45-\mathrm{mg}$ food pellets from the food cup when the feeder was operated. The four subjects in the operant response condition (01-04) were then trained to press the response bar. Each barpress exceeding $.12 \mathrm{~N}$ delivered a food pellet. The schedule of reinforcement was gradually changed over five sessions to a variable-interval 50-sec (VI 50-sec) schedule.

Discrimination training. During this phase, four subjects in the operant group were reinforced for barpressing according to a multiple VI 50-sec EXT schedule. Light and tone (i.e., $\mathrm{L}+\overline{\mathrm{T}}$ and $\overline{\mathrm{L}}+\mathrm{T})$ were separately correlated with the VI 50 -sec schedule for all four subjects. In order to counterbalance stimulus intensity, the first two subjects (01 and 02$)$ had $\overline{\mathrm{L}}+\overline{\mathrm{T}}$ as the EXT-associated stimulus, while the remaining subjects ( 03 and 04$)$ had $L+T$ as the EXT-associated stimulus. A session consisted of 72 -min presentations of each VI-associated stimulus and 14 2-min presentations of the EXT-associated stimulus. The VI and EXT schedules alternated during each 56-min session.

The subjects in the adjunctive behavior group (A1-A4) were placed in the second chamber. This chamber was yoked to the operant chamber so that when food pellets were delivered to the barpressing subjects, food pellets were also delivered in the yoked chamber at the same time and during the same stimulus condition. In the yoked chamber, barpresses had no programmed effect and the behavior recorded was the licking of the water bottle induced by the intermittent delivery of food pellets. Thus, the adjunctive behavior subjects were exposed to a schedule of food delivery that can be conceptualized as a multiple variable-time 50 -sec EXT. The amount of water consumed during each session was recorded. Twenty sessions were conducted following this procedure in order to establish stable differential responding of both the operant and adjunctive behaviors during the stimuli.
Test for additive summation. Testing involved compounding the light and tone elements that had only. been associated with reinforced responding for the operant subjects or the delivery of response-independent food pellets for the adjunctive subjects. For the first two subjects in each group $(01,02, \mathrm{A1}, \mathrm{A} 2)$, the appropriate summation test stimulus was $L+T$. For the remaining subjects $(03,04, \mathrm{A3}, \mathrm{A4})$, the appropriate summation stimulus was $\overline{\mathbf{L}}+\overline{\mathrm{T}}$. The test stimulus was introduced into the mult VI 50-sec EXT schedule for the operant subjects as the third stimulus (the others being $L+\bar{T}$ and $\bar{L}+T$ ) associated with the VI $50-\mathrm{sec}$ schedule. For the subjects in the adjunctive behavior condition, food pellets were also delivered during the summation test stimulus in addition to $\mathrm{L}+\overline{\mathrm{T}}$ and $\overline{\mathrm{L}}+\mathrm{T}$. As before, the operant subject was yoked to an adjunctive subject that experienced the same distribution of food pellets during the stimuli as the barpressing subjects.

A test session consisted of five 2-min presentations of each of the three reinforcement-associated stimuli and 15 presentations of the EXT-associated stimuli. The VI and EXT schedules alternated during each 60 -min session. Testing was conducted for nine sessions.

\section{RESULTS}

For the subjects in the operant condition, mean barpress rates during each stimulus were calculated in blocks of three sessions. These data are presented in Figure 1. The first two blocks of sessions represent the response rates on the last two blocks of sessions prior to the introduction of the summation test stimulus into the

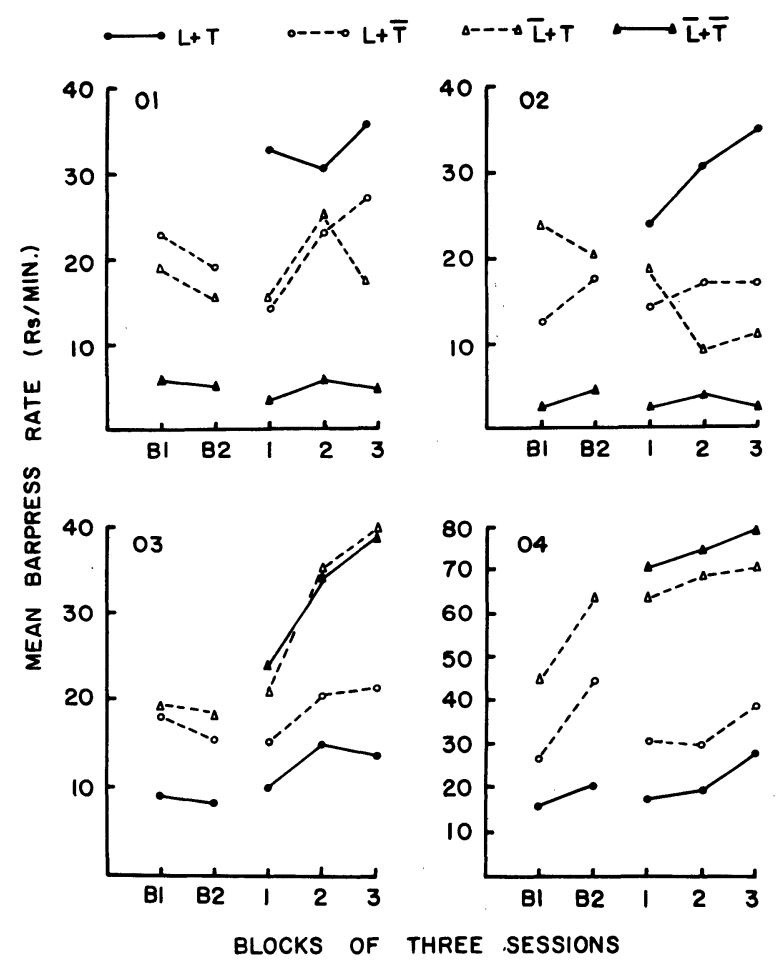

Figure 1. Mean barpressing rates during the stimuli over blocks of three sessions for the subjects in the operant behavior condition. Session Blocks B1 and B2 represent the terminal response rates before the introduction of the summation test stimulus into the multiple schedule. Blocks 1-3 represent response rates during summation testing. 
multiple schedule. This terminal acquisition data shows that the stimuli associated with reinforced barpressing (i.e., $\mathrm{L}+\overline{\mathrm{T}}$ and $\overline{\mathrm{L}}+\mathrm{T}$ ) controlled a higher response rate than the EXT-associated stimulus.

During summation testing (Figure 1, Blocks 1-3) for three of the four subjects (i.e., 01, 02, 04), the summation test stimulus controlled the highest barpress rate of all stimuli over all testing blocks. For Subject 03 the summation test stimulus controlled the highest response rate on one of the three blocks of testing. Thus, additive summation was demonstrated on 10 of the 12 blocks of summation testing. A t test was also performed on the mean of the subject's barpressing rate during the summation test stimulus and the mean response rate during the stimulus that controlled the next highest response rate over the nine summation testing sessions. The $t$ test indicated that the summation test stimulus controlled the highest mean barpress rate of all stimuli $[t(8)=6.14, p<.01]$. Thus, both the analysis of the individual subject's response rates over blocks and the statistical analysis confirmed that additive summation was reliably observed with a schedule-controlled operant behavior.

Session water intake (in milliliters) is presented in Table 1 for the adjunctive behavior group. Mean water intakes are shown for the four baseline sessions, the six terminal acquisition sessions, and for the nine sessions of summation testing. All animals showed schedule-induced polydipsia prior to summation testing and maintained polydipsic levels of water intake during testing, except Subject A1. Subject A1 consumed about $13 \mathrm{ml} /$ session prior to testing, but reduced total session intake to only $10.2 \mathrm{ml}$ during the summation testing.

Figure 2 shows the mean lick rates during the stimuli calculated in blocks of three sessions for the subjects (A1-A4) in the adjunctive behavior group. On the last two blocks of sessions (Blocks B1 and B2), Subjects A1-A3 emitted a higher rate of licking during the stimuli associated with the delivery of food pellets (i.e., $\mathrm{L}+\overline{\mathrm{T}}$ and $\overline{\mathrm{L}}+\mathrm{T}$ ) than during the stimulus during which no food pellets were delivered. Subject A4 exhibited an unusual pattern of licking. The subject consistently emitted a higher rate of licking during the stimulus associated with the absence of food pellets (i.e., $\mathrm{L}+\mathrm{T}$ ) than during the stimuli during which food pellets

Table 1

Mean Water Intake in Milliliters for the Adjunctive Behavior Group During Baseline, Terminal Acquisition (Blocks B1-B2), and Summation Testing (Block 1-3) Phases

\begin{tabular}{cccc}
\hline & & \multicolumn{2}{c}{ Block } \\
\cline { 3 - 4 } Subject & Baseline & B1-B2 & $1-3$ \\
\hline A1 & 6.6 & 13.1 & 10.2 \\
A2 & 4.4 & 17.2 & 14.2 \\
A3 & 6.3 & 19.8 & 17.0 \\
A4 & 3.7 & 19.0 & 21.3 \\
Mean & 5.3 & 17.3 & 15.7 \\
\hline
\end{tabular}

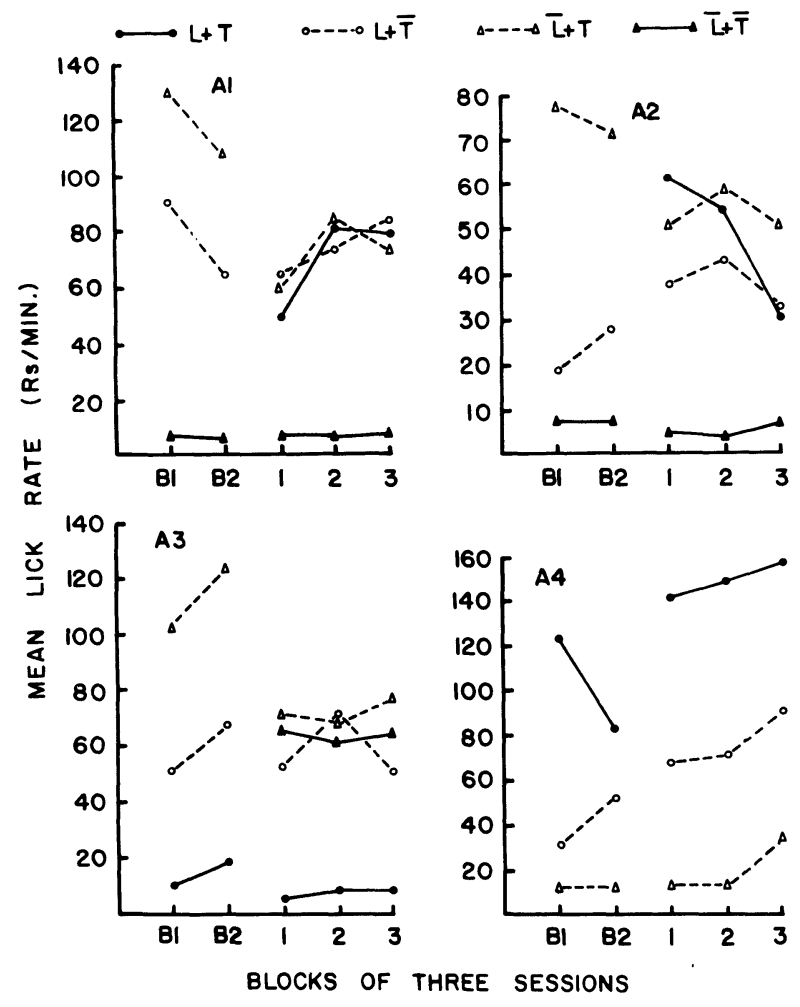

Figure 2. Mean licking rates during the stimuli over blocks of three sessions for the subjects in the adjunctive behavior condition. Session Blocks B1 and B2 represent the terminal response rates before the introduction of the summation test stimulus. Blocks 1-3 represent response rates during summation testing.

were delivered. During summation testing (Blocks 1-3), only Subject A2 showed additive summation and only on one block of sessions (Block 1). Thus, none of the four subjects in the adjunctive behavior condition showed reliable additive summation. A t test on the mean lick rate during the summation test and the lick rate during the stimulus that controlled the next highest (or highest) lick rate was not significant. Thus, additive summation was not demonstrated with an adjunctive behavior.

\section{DISCUSSION}

According to Weiss' (1972) attentional process model of summation, additive summation is the result of the summation test stimulus being a compound stimulus composed exclusively of stimulus elements that either (1) are discriminative for a response rate increase or (2) signal an increase in reinforcement frequency. With the exception of Subject A4, whose licking pattern was atypical, the operant and adjunctive behavior data (Figures 1 and 2 , respectively) show that $\mathrm{L}+\overline{\mathrm{T}}$ and $\overline{\mathrm{L}}+\mathrm{T}$ each were associated with higher response rates than the stimulus associated with no food. Thus in both the adjunctive and operant behavior conditions, the summation test stimulus was exclusively comprised of stimulus elements that appeared to be discriminative for a response rate increase. According to Weiss' model this should be sufficient to produce additive summation of both the operant and adjunctive behaviors. However, the 
results of the experiment demonstrated that additive summation was only obtained with the operant behavior, thus suggesting that the adjunctive drinking was not under discriminative control of the stimuli.

Food pellets were only delivered during the $\mathrm{L}+\overline{\mathrm{T}}$ and $\overline{\mathrm{L}}+\mathrm{T}$ for both the operant and adjunctive subjects, and not during $\overline{\mathrm{L}}+\overline{\mathrm{T}}$ (for Subjects 01, 02, A1, A3) or $\mathrm{L}+\mathrm{T}$ (for Subjects 03, 04, A3, A4). Thus, the summation test stimulus was comprised exclusively of stimulus elements that signaled an increase in the frequency of food delivery. Consequently, Weiss' (1972) model again predicts additive summation of both the adjunctive and operant behaviors. However, as before, additive summation was only obtained with the operant response.

The failure to find additive summation with an adjunctive behavior indicates that the underlying processes controlling operant and adjunctive behaviors may differ and supports Falk's (1971) proposal that adjunctive behaviors represent a distinct behavioral class. Thus, while both barpressing and drinking were dependent on the presence of a food pellet, the compounded stimuli acquired discriminative control of barpressing but not of drinking. Other studies have also failed to demonstrate stimulus control over schedule-induced polydipsia (Allen \& Porter, 1977; Allen, Porter, \& Arazie, 1975; Falk, 1969; McCoy \& Christian, 1976; Porter, Arazie, Holbrook, Cheek, \& Allen, 1975). Taken together, the results of the present study and previous studies support Falk's (1971) contention that adjunctive behaviors may represent a behavioral class distinct from operant and respondent behaviors.

\section{REFERENCES}

Allen, J. D., \& Porter, J. H. Sources of control over schedule-induced drinking produced by second-order schedules of reinforcement. Physiology and Behavior, 1977, 18, 853-863.

Allen, J. D., Porter, J. H., \& Arazie, R. Schedule-induced drinking as a function of percentage reinforcement. Journal of the Experimental Analysis of Behavior, 1975, 23, 223-232.

CoHEN, I. L. The reinforcement value of schedule-induced drinking. Journal of the Experimental Analysis of Behavior, 1975, 23, 37-44.

DE VILliers, P. A. Choice in concurrent schedules and a quanti- tive formulation of the law of effect. In W. K. Honig \& E. R. Staddon (Eds.), Handbook of operant behavior. Englewood Cliffs, N.J: Prentice-Hall, 1977.

Falk, J. L. Conditions producing psychogenic polydipsia in animals. Annals of the New York Academy of Science, 1969, 157, 569-593.

FALK, J. L. The nature and determinants of adjunctive behavior. Physiology and Behavior, 1971, 6, 577-587.

FALK, J. L. The origin and functions of adjunctive behavior. Animal Learning \& Behavior, 1977, 5, 325-335.

Herrnstein, R. J. Doing what comes naturally: A reply to Professor Skinner. American Psychologist, 1977, 32, 1013-1016.

McCoy, J. F., \& Christian, W. P. Schedule-induced drinking and reinforcement omission. Physiology and Behavior, 1976, 17, 537-539.

Porter, J. H., \& Allen, J. D. Schedule-induced polydipsia contrast in the rat. Animal Learning \& Behavior, 1977, 5, 184-192.

Porter, J. H., Arazie, R., Holbrook, J. W., Cheek, M. S., \& Allen, J. D. Effects of variable and fixed secondorder schedules on schedule-induced polydipsia in the rat. Physiology and Behavior, 1975, 14, 143-149.

REYNolds, G. S. Behavioral contrast. Journal of the Experimental Analysis of Behavior, 1961, 4, 57-71.

SkINNER, B. F. Herrnstein and the evolution of behaviorism. American Psychologist, 1977, 32, 1006-1012.

Staddon, J. E. R. Behavioral competition in conditioning situations: Notes toward a theory of generalization and inhibition. In H. Davis \& H. M. B. Hurwitz (Eds.), Operant-Pavlovian interactions. Hillsdale, N.J: Lawrence Erlbaum, 1977.

Staddon, J. E. R., \& Simmelhag, V. L. The "superstition" experiment: A re-examination of its implications for the principles of adaptive behavior. Psychological Review, 1971, 78, 3-43.

Weiss, S. J. Stimulus compounding in free-operant and classical conditioning: A review and analysis. Psychological Bulletin, 1972, 78, 189-280.

(Received for publication May 5, 1978.) 